\title{
Projeto acadêmico socorrista em ação: atividade de promoção de saúde as vítimas de desastres naturais em Teresina - PI: relato de experiência
}

Academic rescuer project in action: health promotion activity for victims of natural disasters in Teresina - PI: experience report

Proyecto de salvador académico in acción: actividad de promoción de la salud para víctimas de desastres naturales in Teresina - PI; informe de experiencia

Germano Soares Martins ${ }^{1 *}$, Olívio Ferreira da Silva Júnior ${ }^{2}$, Fabricia Castelo Branco de Andrade Brito ${ }^{1}$, Suênia Maria da Silva Lima ${ }^{3}$, Cássia dos Santos Silva ${ }^{4}$, Anna Karollyna Sousa Silva ${ }^{3}$, Pamella Judith Carvalho Borges ${ }^{5}$, Allexya Ribeiro e Silva ${ }^{2}$, Eva Larissa de Sousa Rocha ${ }^{2}$, Renan Rodrigues Ferreira Lima².

\section{RESUMO}

Objetivo: Relatar e descrever as ações desenvolvidas por alunos das áreas de enfermagem e odontologia, em atividade de apoio as vítimas de desastres naturais na cidade de Teresina-PI. Relato de Experiência: Em março de 2019, a cidade de Teresina capital do estado Piauí foi acometida por fortes chuvas que deixaram muitos estragos na população que vivia as margens de córregos, riachos e lagos, revelando um grande problema que faz parte da rotina da sociedade contemporânea, principalmente nas cidades onde a expansão não se dá de forma planejada. Esse evento de causas naturais trouxe grandes impactos na vida das pessoas acometidas, e despertou a solidariedade das pessoas, entretanto faltou uma atividade que envolvesse a saúde e o social, e a partir dessa situação surgiu o desejo e o projeto acadêmico socorrista em ação, que organizou e viabilizou essa atividade. Considerações Finais: A experiência vivenciada permitiu aos estudantes refletirem sobre a adequação das ações de serviços segundo a situação social vivida e a necessidade de cada população.

Palavras-chave: Desastres naturais, Socorro de urgência, Odontologia comunitária, Atenção à saúde, Enfermagem em saúde comunitária.

\begin{abstract}
Objective: To report and describe the actions developed by students in the areas of nursing and dentistry, in support activities for victims of natural disasters in the city of Teresina-PI. Experience Report: In March 2019, the city of Teresina, capital of the state of Piauí, was hit by heavy rains that left much damage to the population that lived along the banks of streams, streams and lakes, revealing a major problem that is part of the routine of contemporary society, especially in cities where expansion is not planned. This event of natural causes brought great impacts on the lives of the people affected, and aroused the solidarity of the people, however there was a lack of an activity that involved health and the social, and from this situation arose the desire and the academic first aid project in action, which organized and made this activity feasible. Final Considerations: The experience allowed students to reflect on the adequacy of service actions according to the social situation experienced and the needs of each population.
\end{abstract}

Keywords: Natural disasters, Emergency relief, Community dentistry, Health care, Community health nursing.

\footnotetext{
${ }^{1}$ Faculdade UNINASSAU (Aliança), Teresina - PI. *E-mail: germano140@hotmail.com

${ }^{2}$ Centro Universitário UNIFACID/WYDEN, Teresina - PI.

${ }^{3}$ Faculdade Estácio de Sá, Teresina - PI.

${ }^{4}$ Centro Universitário UNINOVAFAPI, Teresina - PI.

${ }^{5}$ Faculdade UNISULMA, Imperatriz - MA.
} 


\section{RESUMEN}

Objetivo: Informar y describir las acciones desarrolladas por los estudiantes en las áreas de enfermería y odontología, en actividades de apoyo a víctimas de desastres naturales en la ciudad de Teresina-PI. Informe de Experiencia: En marzo de 2019, la ciudad de Teresina, capital del estado de Piauí, fue golpeada por fuertes lluvias que dejaron mucho daño a la población que vivía a lo largo de las orillas de arroyos, arroyos y lagos, revelando un problema importante que es parte de la rutina de la sociedad contemporánea, especialmente en ciudades donde la expansión no está planeada. Este evento de causas naturales trajo grandes impactos en la vida de las personas afectadas, y despertó la solidaridad de las personas, sin embargo, faltaba una actividad que involucrara la salud y lo social, y de esta situación surgió el deseo y el proyecto académico de primeros auxilios en acción, que organizó e hizo posible esta actividad. Consideraciones finales: La experiencia vivida permitió a los estudiantes reflexionar sobre la idoneidad de las acciones de servicio de acuerdo con la situación social experimentada y las necesidades de cada población.

Palabras clave: Desastres naturales, Socorro de urgencia, Odontologia comunitaria, Atencíon a la salud, Enfermería em salud comunitária.

\section{INTRODUÇÃO}

Os desastres são situações que podem ocorrer em todos os lugares do planeta, sendo que alguns lugares apresentam maior vulnerabilidade, estes podem trazer consequências em todos os contextos do desenvolvimento de uma comunidade, região ou país. Tal situação pode influenciar diretamente nos serviços de saúde principalmente no que diz a capacidade e habilidade de atendimento, além dos prejuízos somados a economia, ao social e cultural, e em sua maioria ao meio ambiente (ROCHA MW, 2018).

O que se tem visto na sociedade contemporânea é o avanço exacerbado das cidades, os processos industriais cada vez mais complexos, fato estes que revelam uma situação alarmante no que diz respeito à destruição de recursos naturais, a partir da relação progresso/meio ambiente, surge os problemas relacionados aos desastres naturais que passaram a ser cada vez mais comum (SANTOS PZ, et al. 2019).

Já para Freitas CM, et al. (2014) os desastres naturais são resultados de uma combinação de determinantes importantes para a saúde coletiva que compreende-se como: a ameaça natural propriamente dita, o quão exposta está a população, a vulnerabilidade da mesma e a capacidade preventiva ou de redução de danos que a situação pode oferecer a população, assim estes eventos afetam a estrutura social das comunidades e de forma bastante significativa altera a organização socioambiental destes.

A repercussão causada pelos desastres naturais ou ambientais, exercem força suficiente que leva a certa traumatização da vida das pessoas que passam por essa situação. No Brasil o que se tem visto é um aumento e intensidade cada vez maior desses eventos, o que revela a necessidade de criar-se uma base comum que fortaleça atenção à saúde de forma integral e multiprofissional priorizando o bem-estar dessas vítimas (PEREIRA LC, et al., 2019).

Em geral a resposta aos desastres naturais é dada em circunstâncias conturbadas e em acentuada complexidade, situação que faz com que necessitem de ações que contem com a participação conjunta dos diferentes setores da sociedade, fator esse que envolve geralmente pessoas e produtos que estão geograficamente distantes o que pode dificultar dois aspectos principais o da comunicação e coordenação, que devem ser feitos de forma efetiva para que proporcione bem estar imediato as vítimas (CARDOSO D, et al., 2014).

$\mathrm{Na}$ ótica da saúde pública os desastres são considerados como um grande desafio seja pelos danos humanos, que em geral faz vítimas fatais, ou provoca lesões que levam a enfermidades que geram impacto aos serviços de saúde a curto e médio e longo prazo, como também revela a capacidade de respostas desses serviços dada as características da demanda, que em suma apresentam-se precária, outros fatores que implicam ainda mais é o desabastecimento de água, luz e a dificuldade acesso das equipes de apoio (SOBRAL A, et al., 2010). 
A partir dessa perspectiva a promoção da saúde pode ser definida como uma atividade de capacitação das comunidades, para que auxiliem na atuação e melhoria da qualidade de vida e saúde, sendo priorizado a eles princípios básicos como o da equidade, solidariedade, democracia e entre outros que possam contribui com o bem estar (MACHADO MFAS, et al., 2007).

Dentro da temática da promoção da saúde, encontram se também uma importante aliada que é a Educação em Saúde, que a partir de bases solidas e preestabelecidas atua com o intuito de potencializar a participação dos indivíduos em sua comunidade, corroborando com a melhoria das condições de saúde do meio em que vivem (FEITOSA ALF, et al., 2019). Assim esse estudo como objetivo relatar e descrever as ações desenvolvidas por alunos das áreas de enfermagem e odontologia, em atividade de apoio as vítimas de desastres naturais na cidade de Teresina - PI.

\section{RELATO DA EXPERIÊNCIA}

Em março de 2019, a cidade de Teresina capital do estado Piauí foi acometida por fortes chuvas que deixaram muitos estragos na população que vivia as margens de córregos, riachos e lagos, revelando um grande problema que faz parte da rotina da sociedade contemporânea, principalmente nas cidades onde a expansão não se dá de forma planejada.

Esse evento de causas naturais trouxe grandes impactos na vida das pessoas acometidas, e despertou a solidariedade das pessoas, entretanto faltou uma atividade que envolvesse a saúde e o social, e a partir dessa situação surgiu o desejo e o projeto acadêmico socorrista em ação, que organizou e viabilizou essa atividade na qual se de descreve a seguir.

A atividade foi organizada por uma instituição privada de ensino superior da cidade, que ao ver a situação da comunidade atingida, sensibilizou a comunidade acadêmica dos cursos de enfermagem e odontologia para realização da mesma.

$\mathrm{Na}$ fase de planejamento da atividade que teve celeridade no processo, dada a urgência da situação, foi necessária uma visita prévia a comunidade para fazer um levantamento das áreas mais afetadas e em que direção seria realizada a atividade a fim de contemplar as maiores necessidades.

A visita pela comunidade foi orientada e guiada por Agentes Comunitários de Saúde (ACS) da Unidade Básica de Saúde (UBS) que atende a área, eles conduziram os professores e alunos coordenadores, mostrando os pontos mais atingidos e levantando considerações sobre as necessidades mais pertinentes da comunidade.

A partir disso ficou determinado um ponto de apoio onde seria mais fácil reunir o maior número de pessoas, o local escolhido foi à praça local/central da comunidade, que precisou de autorização da Superintendência de Desenvolvimento Urbano Leste (SDU-LESTE), órgão esse vinculado a prefeitura, outro apoio que se mostrou pertinente foi o da paróquia do bairro.

Marcada a data da ação foi desenvolvida em dois eixos, o primeiro voltado para arrecadação de alimentos não perecíveis e donativos como: colchões, roupas, água potável e material de higiene pessoal, e o segundo foi voltado para avaliação das condições de saúde, onde foi realizado ações voltadas para necessidades já pré-estabelecida no momento da primeira visita. As duas equipes a de enfermagem e a de odontologia foram dividias e ficaram na linha de frente de ação, contemplando diferentes eixos dessa atividade.

A equipe de enfermagem ficou responsável pelas ações que avaliaram as condições de saúde, como a verificação de pressão arterial sistêmica, verificação de glicemia capilar, condições de higiene e orientações quanto à higienização de alimentos e cuidados com o próprio corpo, além disso, realizou-se visitas domiciliares onde foram verificados condições de saúde de idosos acamados e crianças, nessas visitas era priorizado a avaliação das condições básicas de saúde, nos casos que foram necessários foi indicado a busca pelo serviço de saúde. Já a equipe de odontologia foi direcionada para triagem das pessoas que seriam atendidas, organização e distribuição de senhas para entrega de alimentos e roupas. 
Outra importante atividade realizada foi uma oficina para crianças e adultos sobre a correta escovação dos dentes, onde também foram distribuídos kits de higiene bucal para ambos, o kit básico era composto de creme dental, fio dental, escova, já o para adultos portadores de dentadura era disponibilizado de forma extra uma escova especial própria para essa finalidade, bem como um estojo para guardar a prótese.

A atividade foi realizada em consonância com as diretrizes da instituição para atividades desse tipo e com supervisão dos docentes de cada curso, e teve bastante adesão por parte da comunidade, gerando bons comentários, deve-se isso, à importância dada a eles nesse momento delicado, onde empatia e solidariedade contam muito e agregam valores.

\section{DISCUSSÃO}

As situações de desastres ainda se constituem como um grande desafio para o atendimento efetivo e ágil, sendo cada vez mais pertinente o preparo das instituições de saúde para atuação no momento exato que essas situações eclodirem, para tanto faz-se necessário que a gestão local de saúde elabore planos de ação, considerando as vulnerabilidades, e a capacidade de atendimento do serviço, estes planos devem ser bem definidos e cristalinos, e devem atender de forma integral todos os âmbitos da saúde da população, compondo em seu escopo a multidisciplinaridade dos atendimentos de saúde (SILVA MA e CARVALHO R, 2003).

$\mathrm{Na}$ atualidade assegurar que a população tenha um nível elevado de saúde é constituído como um desafio, dadas as condições globais e as mudanças que a sociedade sofre desde as revoluções industriais e tecnológicas mais recentemente, grande ações ao longo dos anos como os controles de pandemias modificaram através da mudança de hábitos e reconhecimento de riscos o comportamento das pessoas em relação à saúde (DIAS S e GAMA A, 2014).

A promoção de saúde deve estar inserida nos mais variados espaços da sociedade, seja ela para prevenção de agravos, promoção de saúde, ou reabilitação, sempre adotando metas que visem promover a integridade das condições de saúde da população em sua coletividade.

Neste contexto deve-se compreender que a saúde e tem que chegar ao ambiente que à comunidade está mais necessitada, assim podendo-se adequar a realidade e traçar uma segura abordagem que respeite tanto os saberes técnicos como os empíricos (PEREIRA-JÚNIOR LA e BERETTA RCS, 2019).

Para discutirmos adequadamente sobre a temática da promoção de saúde através das ramificações da educação em saúde, é necessário que entendamos o complexo saúde-doença, tomando como base que este é resultante dos modos de convivência social e as influências sobre os indivíduos, tais como a instrução de ensino, os aspectos culturais, a intelectualização, além dos ligados ao meio ambiente onde vivem e as situações econômicas, situações essas que se fazem corriqueiras nos determinantes do bem-estar em saúde (RANGEL SM, et al., 2013).

Nessa perspectiva dentro da construção de um indivíduo autônomo capaz de proporcionar seu próprio cuidado, entra a educação em saúde, que pode ser compreendida como parte de um processo educativo que propicia conhecimento dentro da temática da saúde, neste sentido cabe ao educador em saúde elencar as necessidades de cada situação e construir um planejamento que contribua com o conhecimento prévio da comunidade e além de melhorar fortalecer as condições de saúde dos envolvidos, nesse processo que pode ser definido mais como uma troca de saberes (MOREIRA MJ, et al., 2019).

Em uma visão geral das condições de saúde da população, deve-se da ênfase também a promoção da saúde bucal, a qual transcende os ramos técnicos do campo da odontologia, sendo está inserida nas demais praticas que promovem a o fortalecimento da saúde coletiva, visto que a partir do reconhecimento precoce de riscos e problemas relacionados a saúde bucal e consequente resolutividade é possível manter o estado pleno de saúde do indivíduo (REIS DM, et al., 2010).

Ao conceituarmos saúde bucal, devemos compreender suas práticas que visam em geral promover, recuperar e manter a qualidade dos tecidos e estruturas anatômicas associados a cavidade oral em perfeito estado de saúde. 
Na perspectiva da saúde coletiva deve se atentar não apenas para o processo mórbido, mais a busca ativa e identificação precoce de possíveis alterações, que podem servir como base para o reconhecimento de problemas de certa comunidade, assim alertando os profissionais para o desenvolvimento de ações concretas para redução de processos patológicos e para reabilitação da saúde (PREUS RA, et al., 2019).

Nesta temática faz-se pertinente enfatizar que a conexão entre os sistemas de ensino-serviços de saúdecomunidade, pode proporcionar uma abordagem que se explana ao reconhecimento da realidade de uma determinada comunidade, no entanto para que haja uma construção crítica de considerações a respeito de determinada população é necessário a execução de ações que promovam a saúde de maneira consciente, integrativa e efetiva, assim através da aproximação do saber teórico e a prática, pode se afirmar que o conhecimento de realidades auxilia no correto planejamento e desenvolvimento de atividades onde a intervenção propicia números benefícios aos atendidos (VENDRUSCOLA C, et al., 2017).

Portanto a partir da construção desse estudo e seguindo sua cronologia, faz-se fundamental que a saúde seja compreendida como um determinante de qualidade de vida e como condição básica para o bom exercício das atribuições cidadãs dos indivíduos atendidos, assim a quebra de barreiras e estigmas em relação aos serviços deve ser feita de forma a conscientizar a população, pois a partir daí temos um serviço de boa qualidade, humanizado e com efetividade no seu atendimento, fornecendo, segurança e a garantia do retorno dos pacientes (DATO CD, et al., 2019).

A experiência vivenciada permitiu aos estudantes refletirem sobre a adequação das ações de serviços de saúde segundo a situação social vivida e a necessidade individual de cada comunidade. Neste sentido, a vivência permitiu, ainda, aos acadêmicos exercerem práticas de cidadania e solidariedade, ao levarem conforto neste momento de dificuldade, pelo qual as famílias passavam.

Com isto, tais práticas suscitam o enriquecimento na formação do perfil do egresso, de forma crítica, humanizada e transformadora, contribuindo assim com a sociedade, ficando cristalino o comprometimento e responsabilidade social dos envolvidos na atividade.

\section{REFERÊNCIAS}

1. CARDOSO D, et al. Gestão de conhecimento nas respostas a desastres naturais: a experiência da defesa civil do estado de Santa Catarina. Perspectivas em Gestão \& Conhecimento, 2014; 4(2):90-106.

2. DATO CD, et al. A busca pela humanização da assistência na educação permanente em saúde, Revista da Enfermagem em Evidencia, 2019; 3(1): 224-238.

3. DIAS S, GAMA, A. Promoción da saúde: evolução de um paradigma, desafios contemporâneos. Rev. Salud Pública, 2014; 16(2): 307-317.

4. FEITOSA ALF, et al. Sala de espera: estratégia de educação em saúde no contexto da atenção básica. Rev. Bra. Educ. Saúde, 2019; 9(2): 67-70.

5. FREITAS CM, et al. Desastres naturais e saúde: uma análise da situação do Brasil. Ciência \& Saúde Coletiva, 2014; 19(9): 3645-3656.

6. MACHADO MFAS, et al. Integridade, formacão de saúde, educação em saúde e as propostas do SUS-uma revisão conceitual. Ciência \& Saúde Coletiva, 2007; 12(2): 335-342.

7. MOREIRA MJ, et al. Educação em saúde no ensino de graduação em enfermagem. Rev. Enferm Atenção Saúde, 2019; 8(1): $61-10$

8. PEREIRA LC, et al. Assistência de enfermagem em situações de desastres ambientais. Revista Multidisciplinar do Nordeste Mineiro, 2019; 2 .

9. PEREIRA-JÚNIOR LA, BERETTA RCJ. A educação em saúde como ferramenta para uma formação profissional multidisciplinar. Revista Eletrônica Califori, 2019; 3(1).

10. KALCHRAIBER FC, et al. Pedagogical strategy for teaching and learning epidemiology in nursing undergraduate. Revista Brasileira de Enfermagem, 2019; 72(2):414-9.

11. PREUS RA, et al. Problemas de saúde bucal, formas de controle sob a visão da saúde coletiva e tratamento multidisciplinar. Revista Faipe, 2019; 9(2): 70-82.

12. RANGEL, SM, et al. Ação extensinonista de acadêmicos de enfermagem em escola pública: educação e promoção em saúde na comunidade. Rev. Enfermagem Revista, 2013; 16(2).

13. REIS DM, et al. Educação em saúde como estratégia de promoção de saúde bucal em gestantes. Ciência \& Saúde Coletiva, 2010; 15(1): 269-276.

14. ROCHA MW, Preparação de profissionais de enfermagem para assistência de desastres socioambientais. (Mestrado em Enfermagem) -Escola de Enfermagem Anna Nery, Universidade Federal do Rio de Janeiro, Rio de Janeiro, 2018; $163 f$.

15. SANTOS PZ, et al. Educação permanente sobre a atenção psicossocial em situação de desastres para agentes comunitários de saúde: um relato de experiência. Saúde Debate, 2019; 43(3): 200-208.

16. SILVA MA, CARVALHO R. Situação de desastre: atuação da equipe de enfermagem em cirurgias emergenciais. Rev. SOBECC, 2013; 18(2): 67-76.

17. SOBRAL A, et al. Desastres naturais - sistemas de informação e vigilância: uma revisão da literatura. Epidemiol. Serv. Saúde, 2010; 39(4): 389-402.

18. VENDRUSCOLA C, et al. Integração ensino serviço-comunidade na perspectiva da reorientação da formação em saúde. Revista SUSTINERE, 2017; 5(2): 245-259. 